\title{
Case report - lazarus syndrome after prolonged resuscitation
}

\author{
Sze-Ying Thong ${ }^{1 *}$ and Shin-Yi Ng² \\ *Correspondence: thongszeying@gmail.com \\ 'Department of Anaesthesia Singapore General Hospital College road, Singapore 169608, Block 2 level 2, Singapore. \\ ${ }^{2}$ Singapore General Hospital, College road, Singapore 169608, Block 2 level 2, Singapore.
}

\begin{abstract}
A 62 -year-old male with a history of complete heart block with pacemaker pacing was found unresponsive after a fall whilst inpatient. He sustained severe head injury and was transferred to the Intensive Care Unit. Five minutes after arrival, patient developed ventricular fibrillation. Cardiopulmonary resuscitation commenced. Patient was intubated and his respiratory function supported on ventilator. The predominant cardiac rhythm was ventricular fibrillation. There was a transient (30 seconds) return of pulse 30 minute into the resuscitation. This ventricular tachycardia rapidly degenerated into ventricular fibrillation and then idioventricular rhythm with un-captured pacemaker spikes. Other features of circulatory arrest including absent heart sounds, pulse, blood pressure, and the cessation of breathing. Resuscitation was terminated after another 10 minutes. In total, 15 doses of intravenous adrenaline 1mg; IV lidocaine $75 \mathrm{mg}$; 15 cycles of 360J monophasic defibrillation were delivered during the 40-minute resuscitation. Ventilation was continued until the pacemaker could be deactivated. This idioventricular rhythm with un-captured pacemaker spikes lasted 5 minutes after the termination of unsuccessful resuscitation. The return of spontaneous circulation subsequently occurred with an intrinsic ventricular rhythm of rate of 55-75 beats per minute. Pacemaker spikes were seen, without mechanical capture. Strong regular carotid pulse, previously undetected during ventricular fibrillation, was detected. Noninvasive blood pressure readings and other parameters were listed in table 1. CPR was not restarted and circulation ceased after 34 minutes. After further 124 minutes until the pacemaker was deactivated, patient was certified dead.

Conclusion: Autoresuscitation has implications on the certification of death and the decision to cease resuscitation. Our report illustrates the difficulties in diagnosing cardiovascular death in a patient with 40 minutes of circulatory standstill despite advanced cardiac life support as cardiac function was regained 5 minutes after abandonment of resuscitation. Longer period of time may be required for the cessation of cardiorespiratory function to have occurred in patients with pacemakers before the death can be pronounced.
\end{abstract}

Keywords: Lazarus syndrome, death certification, auto-resuscitation

\section{Background}

Death is understood to have occurred after the irreversible cessation of cardiorespiratory or brain function. However, the physiologic transition from life to death is complex. We describe a patient who had return of spontaneous circulation after cessation of unsuccessful cardiopulmonary resuscitation.

\section{Case}

This patient is a 62-year-old male, with a relevant history of complete heart block and implanted pacemaker pacing. He was admitted to the intensive care unit (ICU) after a fall resulting in an acute drop in consciousness, from Glasgow Coma Scale 15 to 6 , i.e. E1V1M4. Pupils were $3 \mathrm{~mm}$ bilaterally and reactive to light. His vital signs were as follows: blood pressure (BP) 170/90, repeat $260 / 100 \mathrm{mmHg}$, heart rate (HR) $67-80$ beats per minute, respiratory rate (RR) $18-23$ breaths per minute.

Twelve-lead electrocardiogram (EKG) performed in the ICU showed ventricular-paced rhythm rate of 65 and complete heart block (Figure 1). This was similar to his 12-lead EKG on admission to the hospital (Figure 2). Five minutes after the repeat EKG, patient developed ventricular fibrillation, which was observed on the 3-lead monitoring EKG. Cardiopulmonary resuscitation
(CPR) commenced in accordance with the advanced cardiac life support protocol. Patient was intubated and respiratory function supported by the ventilator.

During the entire resuscitation attempt, the predominant cardiac rhythm was ventricular fibrillation with un-captured pacemaker spikes. There was a transient (30 seconds) return of pulse 30 minute into the resuscitation. This ventricular tachycardia seen on the 3-lead EKG rapidly degenerated into ventricular fibrillation and then idioventricular rhythm with un-captured pacemaker spikes. Other features of cardiorespiratory arrest including absent heart sounds, absent pulse, absent blood pressure, and the cessation of breathing were noted.

Resuscitation was terminated after 10 minutes of sustained pulseless idioventricular activity on the EKG. In total, 15 doses of intravenous adrenaline $1 \mathrm{mg}$; IV lidocaine $75 \mathrm{mg} ; 15$ cycles of $360 \mathrm{~J}$ monophasic defibrillation were delivered during the 40-minute resuscitation. Ventilation was continued until the pacemaker could be deactivated.

This pulseless idioventricular rhythm with un-captured pacemaker spikes lasted 5 minutes after the termination of resuscitation. Return of spontaneous circulation occurred subsequently with an intrinsic ventricular rhythm of rate of 

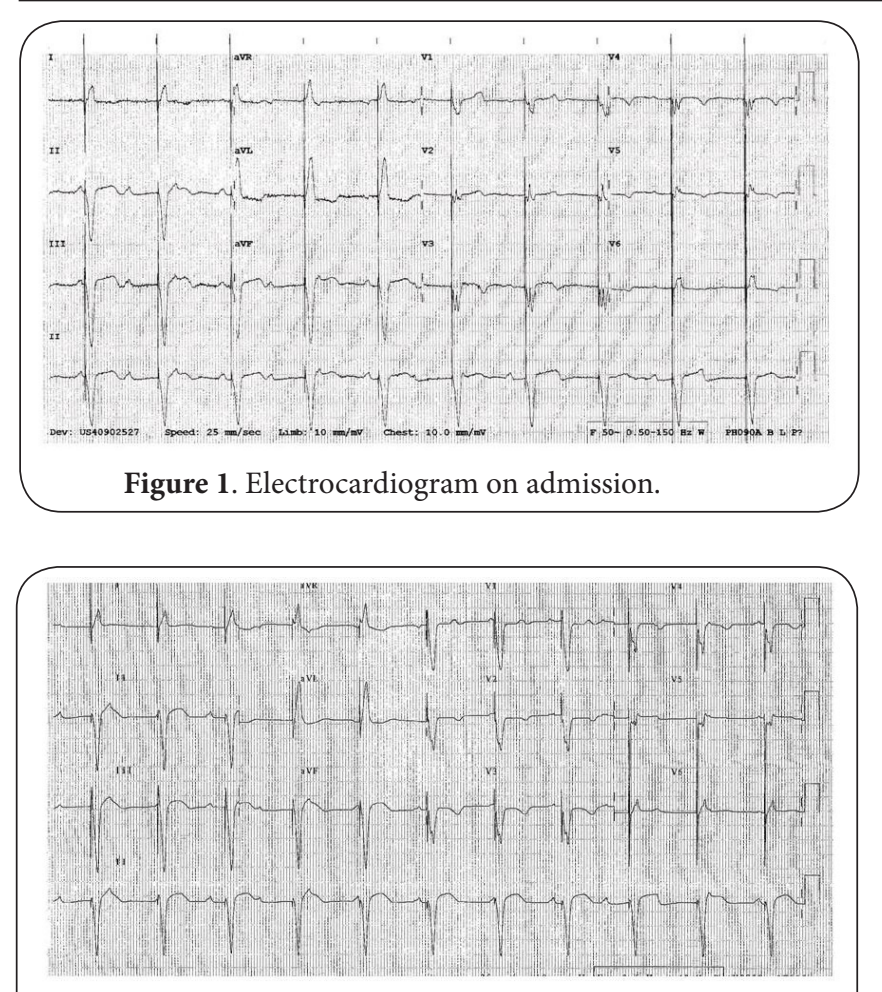

Figure 2. Electrocardiogram just prior to ventricular fibrillation.

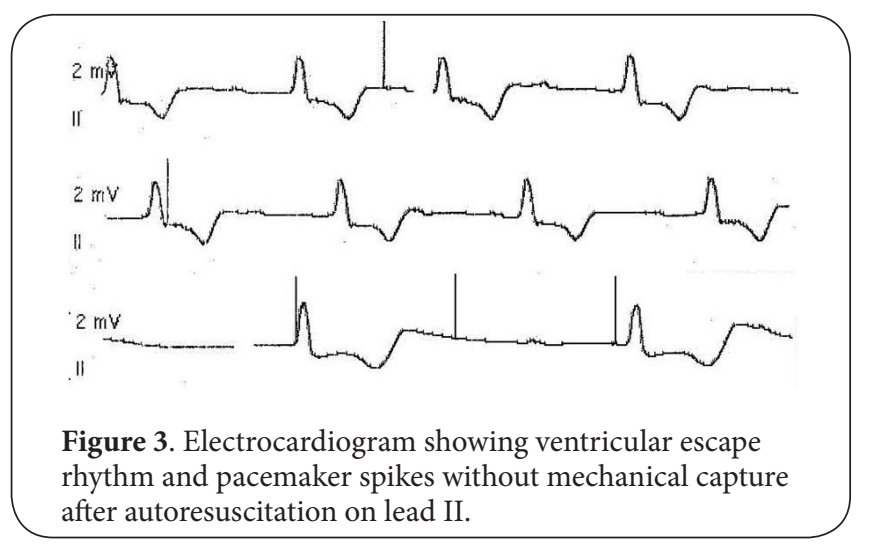

55-75 beats per minute. Pacemaker spikes were seen, without mechanical capture. (Figure 3) Strong, regular carotid pulse, previously undetected during ventricular fibrillation and the idioventricular rhythm, was detected.

Noninvasive blood pressure readings and other parameters were listed in Table 1. Inotropic support was not commenced and circulation ceased after 34 minutes. After a further period of 124 minutes, the pacemaker was deactivated and the patient was certified dead.

\section{Discussion}

Autoresuscitation, as defined by the unassisted return of spontaneous circulation after cardiac arrest, is exceedingly
Table 1. Patient's parameters after spontaneous return of circulation.

\begin{tabular}{lcccccccccccc}
\hline Time & 0751 & 0753 & 0755 & 0800 & 0805 & 0810 & 0815 & 0820 & 0825 & 0830 & 0840 & 0850 \\
\hline HR & 47 & 50 & 46 & 56 & 55 & 53 & 47 & 48 & 60 & 53 & 55 & 28 \\
SBP & 132 & 150 & 164 & 145 & 126 & 119 & 106 & 78 & 58 & $* *$ & $* *$ & $* *$ \\
DBP & 71 & 74 & 68 & 72 & 68 & 67 & 55 & 45 & 22 & $* *$ & $* *$ & $* *$ \\
$\mathrm{SpO}_{2}$ & 100 & 100 & 100 & 100 & 100 & 100 & 100 & 100 & 100 & 98 & 90 & $* *$ \\
RR $^{12}$ & 12 & 12 & 12 & 12 & 12 & 12 & 12 & 12 & 12 & 12 & 12 \\
etCO & 19 & 21 & 22 & 28 & 24 & 30 & 30 & 30 & 27 & 20 & 17 & 10 \\
\hline
\end{tabular}

HR. Heart rate/ beats per minute

SBP. Systolic blood pressure/ $\mathrm{mmHg}$

DBP. Diastolic blood pressure/ $\mathrm{mmHg}$

$\mathrm{SpO}_{2}$. Pulse oximeter venous saturation/ \%

RR. Respiratory rate (triggered by ventilator)/ breaths per minute et $\mathrm{CO}_{2}$. End tidal carbon dioxide/ $\mathrm{mmHg}$

** Indicates unrecordable parameter.

rare. It was first described by Linko et al., and subsequently called “Lazarus phenomenon" by Bray $[1,2]$. The asystolic heart restarts and is able to sustain contractile activity necessary to perfuse the vital organs [3].

The circulatory system is a close loop of blood vessels in series with the heart, which contracts drives blood flow. The smooth muscles in the blood vessels actively regulate the resistance and capacitance of this closed system, generating a perfusion pressure. Even during asystole, this perfusion pressure can persist in the coronary and cerebral vessels, providing a plausible explanation for recovery after autoresuscitation [4].

In this patient, cardiorespiratory arrest occurred as evidence by the absent heart sound, absent pulse, absent blood pressure, and the cessation of breathing. This period of "death" lasted for five minutes after the cessation of cardiopulmonary resuscitation for ventricular fibrillation lasting forty minutes. It may be possible that the pacemaker activity triggered patient's cardiac electrical activity. However, recorded strips of 3-lead EKG showed very infrequent pacemaker spikes and the lack of mechanical capture, which should follow, if that was the case. The rhythm after autoresuscitation was an irregular ventricular escape rhythm.

There is only one case report of autoresuscitation possibly triggered by a pacemaker in literature. Weiss et al., reported a patient who suffered cardiac arrest during surgery. Due to poor prognosis, cardiopulmonary resuscitation was not initiated and the high dose catecholamine infusion was terminated. However, his pacemaker continued to function and after 6 minutes of cessation of cardiopulmonary function, circulation restarted spontaneously. Surgery continued but patient died 2 days later in the ICU without regaining consciousness [5].

The mechanism of autoresuscitation remains unknown, but it has been postulated to relate to dynamic lung hyperinflation, excessive intrathoracic pressure and resultant tamponade physiology that may give the false appearance of circulatory arrest [6]. However, this explanation fails to explain the very reason CPR was initiated in this patient - ventricular fibrillation with cardiac arrest. The absence of obstructive lung 
disease also makes auto-PEEP a less likely explanation. Other possible mechanisms suggested included delayed delivery to the heart of previously administered adrenaline; gradual intracellular shift of potassium after hyperkalemic arrest; cardiac reperfusion after spontaneous dislodgement of embolized endovascular plaque from the coronary artery [7].

A systemic review by Hornby et al., in 2010 found 32 cases of autoresuscitation, which occurred between few seconds to 33 minutes after cessation of failed CPR. In studies reporting exact times and appropriate monitoring, autoresuscitation did not occur beyond 7 minutes after CPR [3]. All the studies were considered of being very low quality [8]. It was also uncertain whether some of the reports reflected errors in the diagnosis of "death".

Autoresuscitation has implications on the certification of death, the decision to cease resuscitation efforts and endof-life organ procurement. Increasing medical sophistication and extraordinary life support measures further confound our grasp and understanding of death. Although recommendations exist for diagnostic criteria, there is no consensus on the duration that circulation and respiration must cease for a person to be determined dead $[\mathbf{9 , 1 0}$.

In this report, patient's cardiovascular deterioration, resuscitative efforts, autoresuscitation and eventual irreversible cardiopulmonary arrest were continuously monitored and recorded by multiple monitors, thus providing validity to the claims of Lazarus syndrome. This case illustrates the difficulties in diagnosing cardiovascular death in a patient with 40 minutes of circulatory standstill despite advanced cardiac life support as cardiac function was regained 5 minutes after abandonment of resuscitation.

It is possible that a longer period of time is required for the cessation of cardiorespiratory function in patients with pacemakers before the death can be pronounced. We believe that this case is a significant addition to the limited knowledge of this enigma.

\section{Competing interests}

The authors declare that they have no competing interests.

\section{Authors' contribution}

SYT performed research related to this study, designed and drafted the manuscript.

SYN conceived the idea of reporting this case, edited and approved the anuscript.

Publication history

Received: 23-Dec-2012 Revised: 18-Jan-2013

Accepted: 19-Jan-2013 Published: 16-Feb-2013

\section{References}

1. Linko K, Honkavaara $P$ and Salmenpera M: Recovery after discontinued cardiopulmonary resuscitation. Lancet 1982, 1:106-7. | Article | PubMed

2. Bray JG, Jr.: The Lazarus phenomenon revisited. Anesthesiology 1993, 78:991. | Article | PubMed

3. Hornby K, Hornby L and Shemie SD: A systematic review of autoresuscitation after cardiac arrest. Crit Care Med 2010, 38:1246-53. | Article | PubMed
4. Rady MY and Verheijde JL: Autoresuscitation and determining circulatory-respiratory death in clinical practice for organ donation. Crit Care Med 2012, 40:1655-6. | Article | PubMed

5. Wiese $\mathrm{CH}$, Stojanovic T, Klockgether-Radke A, Bartels U, Schmitto JD, Quintel M and Graf BM: [Another case of "Lazarus phenomenon" during surgery? Spontaneous return of circulation in a patient with a pacemaker]. Anaesthesist 2007, 56:1231-6. I Article I PubMed

6. Rogers PL, Schlichtig R, Miro A and Pinsky M: Auto-PEEP during CPR. An "occult" cause of electromechanical dissociation? Chest 1991, 99:492-3. | Article | PubMed

7. Ben-David B, Stonebraker VC, Hershman R, Frost CL and Williams HK: Survival after failed intraoperative resuscitation: a case of "Lazarus syndrome". Anesth Analg 2001, 92:690-2. I Article I PubMed

8. Guyatt GH, Oxman AD, Vist GE, Kunz R, Falck-Ytter Y, Alonso-Coello P and Schunemann HJ: GRADE: an emerging consensus on rating quality of evidence and strength of recommendations. BMJ 2008, 336:924-6. | Article | PubMed Abstract | PubMed Full Text

9. Curfman GD, Morrissey S and Drazen JM: Cardiac transplantation in infants. N Engl J Med 2008, 359:749-50. | Article | PubMed

10. Bernat JL: The boundaries of organ donation after circulatory death. $N$ Engl J Med 2008, 359:669-71. | Article I PubMed

\section{Citation:}

Thong S-Y and Ng S-Y: Case report - lazarus syndrome after prolonged resuscitation. journal of Anesthesiology and Clinical Science 2013, 2:14.

http://dx.doi.org/10.7243/2049-9752-2-14 